\title{
First Report of Rust Disease Caused by Puccinia nishidana on Cirsium setidens
}

\author{
Mi-Jeong Park ${ }^{1}$, Jun-Keun Choi ${ }^{2}$, Makoto Kakishima ${ }^{3}$ and Hyeon-Dong Shin ${ }^{1 *}$ \\ ${ }^{1}$ Division of Environmental Science and Ecological Engineering, Korea University, Seoul 136-701, Korea \\ ${ }^{2}$ Gangwon Agricultural Research \& Extension Services, Chuncheon 200-150, Korea \\ ${ }^{3}$ Graduate School of Life and Environmental Sciences, University of Tsukuba, Tsukuba, Ibaraki 305-8572, Japan \\ (Received on March 24, 2010; Revised on July 15, 2010; Accepted on July 21, 2010)
}

Cirsium setidens Nakai, endemic to Korea, is a perennial plant belonging to the Compositae. It is distributed mainly in mountainous regions throughout the Korean Peninsula. As young leaves and shoots of the plant are edible as well as medicinal, private gardeners in mountainous villages in Korea have frequently grown it for selfconsumption. Recently, commercial cultivation of this vegetable has developed for supplying fresh produce to urban consumers. In September 2009, severe outbreak of rust disease occurred on $C$. setidens cultivated in an experimental farm of Gangwon Agricultural Research \& Extension Services in Pyeongchang, Korea. The rust infections of the plant have been noticed since 1992 (HDS, unpublished data). The rust fungus produced urediniospores within dark brownish pustules, mostly on the lower surface of the infected

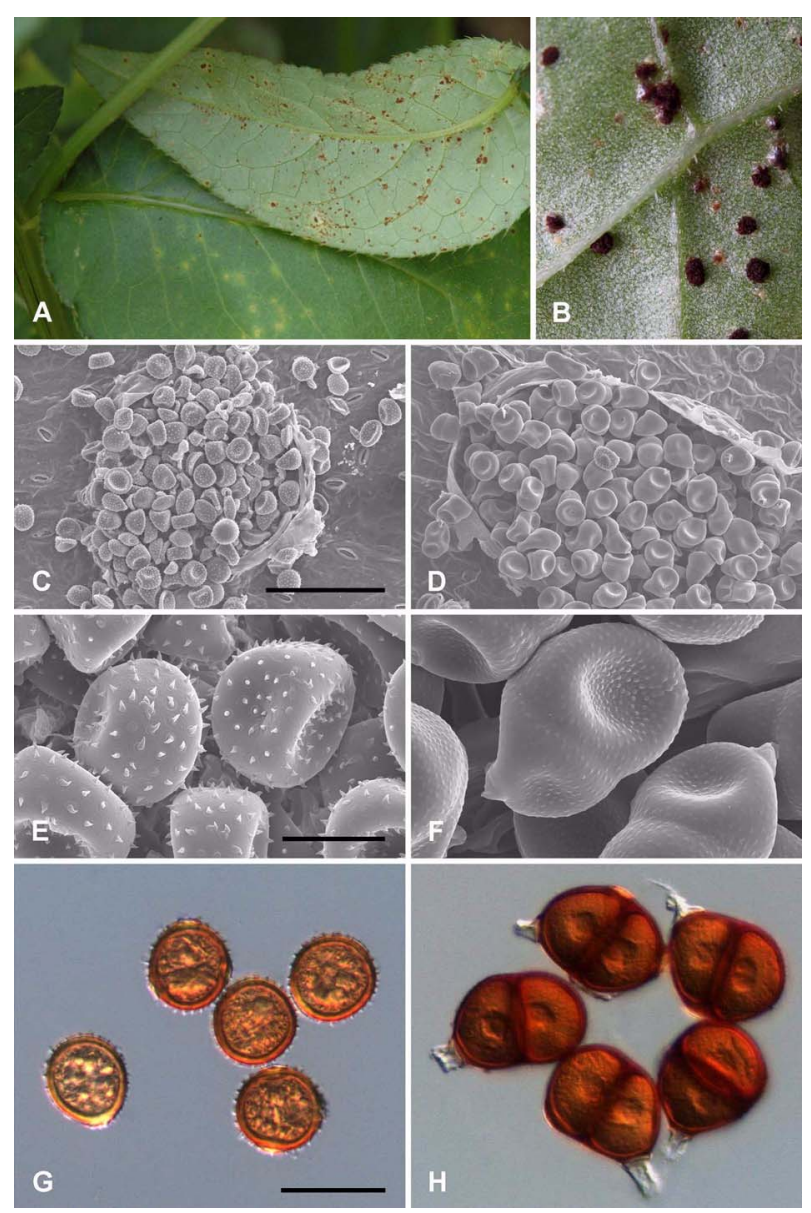

Fig. 1. Symptoms on leaves of Cirsium setidens showing uredinia (A) and telia (B) of Puccinia nishidana. Uredinia (C) and telia (D) as seen by SEM (bar $=100 \mu \mathrm{m})$. Wall ornamentation of urediniospores (E) and teliospores $(\mathrm{F})$ as seen by SEM (bar $=15 \mu \mathrm{m})$. Urediniospores $(\mathrm{G})$ and teliospores $(\mathrm{H})$ as seen by $\mathrm{LM}($ bar $=30 \mu \mathrm{m})$. leaves during summer (Fig. 1A). From August to September, blackish brown pustules bearing teliospores began to produce (Fig. 1B). As the disease progressed, the symptoms of yellowing and necrosis on the leaf lesions became more intense. The voucher specimens were deposited in the herbarium of Korea University, Seoul, Korea.

The morphology of the causal pathogen was examined with LM and SEM. Uredinia were mostly hypophyllous, erumpent, dark brownish, circular to irregular in shape (Fig. 1C). Urediniospores were broadly obovoid to subglobose, yellow-orange to golden brown, echinulate, $25-30 \times 23-25 \mu \mathrm{m}$ (including wall thickness) (Fig. 1E \& G). Telia were mostly hypophyllous, subepidermal at first, later erumpent, blackish brown (Fig. 1D). Teliospores were broadly ellipsoid, chestnut-brown, 35-40×27-30(-33) $\mu \mathrm{m}$, twocelled with slightly verrucose cell walls and a usually broken, colourless pedicel (Fig. 1F \& H). A germ pore of the upper cell was mostly apical, and that of the lower cell was located near septum. There was a slight umbo over each germ pore. Based on these morphological characteristics, this fungus was identified as Puccinia nishidana Henn., as described by Hiratsuka et al. (1992) and Zhuang (2003).

Genomic DNA was extracted from teliospores of the dried herbarium specimens (KUS-F24576, F24870). PCR amplification and sequencing of both ITS2 region of rDNA and D1/D2 region of 28S rDNA were performed using the primers Rust2inv and LR6 as described by Aime (2006). The resulting sequences (approximately $1400 \mathrm{bp}$ ) of the two isolates were identical to each other, and deposited in GenBank (HM022140, HM022141). However, as these were the first sequences for $P$. nishidana, molecular confirmation for the identification could not be made in the present study.

Puccinia nishidana is widely distributed in Japan, China, Russia and Korea (Hiratsuka et al., 1992) and about 30 species of the genus Cirsium have been reported as its host plants, but there has been no previous record of $P$. nishidana on $C$. setidens (Farr and Rossman, 2010). Therefore, this is the first report of rust infection by the fungus on this plant. The occurrence of rust disease on C. setidens will have significant impact on the commercial production of the vegetable and effective control methods will be required.

\section{References}

Aime, M. C. 2006. Toward resolving family-level relationships in rust fungi (Uredinales). Mycoscience 47:112-122.

Farr, D. F. and Rossman, A. Y. 2010. Fungal Databases, Systematic Mycology \& Microbiology Laboratory, ARS, USDA. Retrieved March 12, 2010, from http://nt.ars-grin.gov/fungaldatabases/.

Hiratsuka, N., Sato, S., Kakishima, M., Kaneko, S., Sato, T., Hiratsuka, T., Katsuya, K., Hiratsuka, Y., Ono, Y., Harada, Y. and Nakayama, K. 1992. The Rust Flora of Japan. Tsukuba Shuppankai, Ibaraki, Japan. 1205 pp. $+159 \mathrm{pp}$. (Index)

Zhuang, J. Y. 2003. Flora Fungorum Sinicorum. Vol. 19. Uredinales (II). Science Press, Beijing, China. 324 pp.

\footnotetext{
*Corresponding author (hdshin@korea.ac.kr)
} 